\title{
USE OF NON-QUADRATIC YIELD SURFACES IN DESIGN OF OPTIMAL DEEP-DRAW BLANK GEOMETRY
}

\author{
Roger W. Logan \\ University of California \\ Lawrence Livermore National Laboratory \\ Livermore, $\mathrm{CA}$
}

This paper was prepared for submittal to: 1996 SAE International Congress and Expo

Detroit, MI

February 26-29, 1996

December 1995

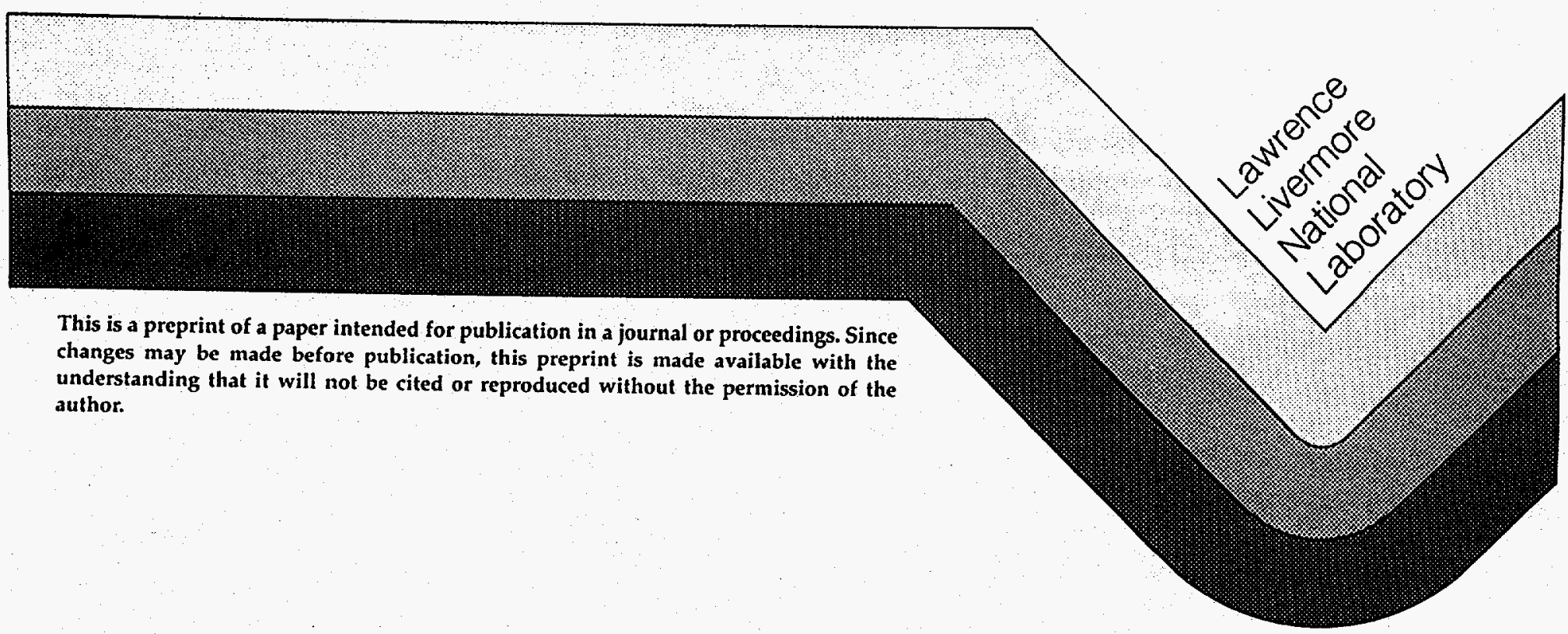




\section{DISCLAIMER}

This document was prepared as an account of work sponsored by an agency of the United States Government. Neither the United States Government nor the University of California nor any of their employees, makes any warranty, express or implied, or assumes any legal liability or responsibility for the accuracy, completeness, or usefulness of any information, apparatus, product, or process disclosed, or represents that its use would not infringe privately owned rights. Reference herein to any specific commercial products, process, or service by tradename, trademark, manufacturer, or otherwise, does not necessarily constitute or imply its endorsement, recommendation, or favoring by the United States Government or the University of California. The views and opinions of authors expressed herein do not necessarily state or reflect those of the United States Government thereof, and shall not be used for advertising or product endorsement purposes. 


\section{DISCLAMMER}

Portions of this document may be illegible in electronic image products. Images are produced from the best available original document. 


\title{
USE OF NON-QUADRATIC YIELD SURFACES IN DESIGN OF OPTIMAL DEEP-DRAW BLANK GEOMETRY
}

\author{
Roger W. Logan \\ University of California \\ Lawrence Livermore National Laboratory
}

\begin{abstract}
Planar anisotropy in the deep-drawing of sheet can lead to the formation of ears in cylindrical cups and to undesirable metal flow in the blankholder in the general case. For design analysis purposes in non-linear finiteelement codes, this anisotropy is characterized by the use of an appropriate yield surface which is then implemented into codes such as DYNA3D. The quadratic Hill yield surface offers a relatively straightforward implementation and can be formulated to be invariant to the coordinate system. Non-quadratic yield surfaces can provide more realistic strength or strain increment ratios, but they may not provide invariance and thus demand certain approximations. Forms due to Hosford and Barlat et al. have been shown to more accurately address the earing phenomenon. In this work, use is made of these non-quadratic yield surfaces in order to determine the optimal blank shape for cups and other shapes using ferrous and other metal blank materials with planar anisotropy. The analyses are compared to previous experimental studies on non-uniform blank motion due to anisotropy and asymmetric geometry.
\end{abstract}

\section{INTRODUCTION AND BACKGROUND}

There are (at least) two primary goals for the engineering analysis of a sheet metal forming process. First, analysis aims to reduce the trial and error in tooling and process design, and thereby reduce material waste and lead times to produce a new part. Second, analysis aims to influence the design of the desired part for ease of manufacture. Both of these goals ultimately lead toward the objective of faster production of better parts at minimum cost. The second goal in particular is usually dependent on material properties of the sheet material, and thus the design analysis may influence either the positioning of the (anisotropic) sheet prior to stamping, or influence the thermo-mechanical processing to provide more desirable forming properties.

One cause of material waste in stamping is the formation of ears on the periphery of the blank from which the part is formed. The formation of ears in simplest form results from the stamping of a circular blank into a cylindrical cup. This phenomenon has been studies both experimentally, and more recently numerically, in numerous works; a partial coverage of these can be found in [1-5].
In both partial [3] and full [4] simulations of the earing phenomenon is cylindrical cups, it was noted that use of the 1948 Hill quadratic anisotropic yield surface [6] is likely to lead to an overprediction of earing. A similar phenomenon was noted with regard to the tendency of the 1948 Hill criterion to overpredict the dependence of the Limiting Draw Ratio (LDR) on the strain ratio $R$. In an effort to overcome these tendencies to overpredict certain deep drawing phenomena, the anisotropic yield criterion suggested in 1979 by Hosford [7] in correlation with crystal plasticity based calculations for fcc metals was implemented into the Lawrence Livermore National Laboratory (LLNL) DYNA3D [8] and used to study the earing phenomenon in [4]. As outlined in that work and below, the 1979 Hosford criterion shows improved correlation to experimental earing observations, but does demand an implementation that is both tedious and numerically approximate in nature, although these drawbacks affect most non-quadratic yield surfaces to some extent. An extension of the 1979 Hosford criterion was suggested by Barlat and Lian in 1989 [9], and with the inclusion of an in-plane shear term this criterion shows added promise for 3-dimensional analyses with a more traditional implementation into finite-element codes.

Thus, the preliminary motivation for this work was to examine, with fully 3-D simulations, the predicted vs. observed earing using both the 1948 Hill quadratic yield criterion and the 1979 Hosford criterion as in [4], and to compare these to the newly implemented TriComponent (1989 Barlat) criterion. At this point, given an expanded suite of anisotropic yield surface capability, our second goal was to study the effect of using (or not using) normal anisotropy and planar anisotropy in determining optimum blank shape for forming processes. We have chosen two part geometries, covering applications of both general and specific interest. In the first, blank shape optimization is studied for the case of square cup or box drawing, and compared to previous experiments [10] on this geometry with a nearly isotropic stainless steel. Next, this same shape will be studied using properties typical of aluminum-killed (AK) drawing quality steel, with a slight adjustment in properties so $R=P$. This in part was done to postpone for a further work the issues arising when $R$ and $P$ are unequal; this situation, not uncommon for Interstitial-Free (IF) steel, warrants a separate discussion, and is mentioned below only in passing. A situation highly typical in LLNL's formability research is the warm forming of unalloyed uranium (designated D38) 
into axisymmetric geometries as discussed below. Even with an axisymmetric geometry, previous works [11] have shown that if the thermomechanical processing is achieved to optimize the LDR, planar anisotropy is induced that may result in earing and thus material waste. Given our great concern about the waste and reprocessing costs for uranium, optimization of the blank shape for this process is highly desirable from an environmental and worker safety standpoint.

\section{YIELD SURFACE IMPLEMENTATIONS}

Building on our previous work with anisotropic yield surfaces [4], we continue here with the Lawrence Livermore National Laboratory (LLNL) version of DYNA3D, our explicit, transient dynamics Lagrangian finite-element code [8]. We find it is usually most efficient to implement a methodology in our explicit DYNA family first, bearing in mind that the formulation must be appropriate for subsequent implementation into the implicit NIKE3D [12] as well. Such is the case in this work, which examines the usefulness of new yield surfaces implemented into an internal W-DYNA version of our DYNA code family. Previously Whirley, Engelmann, and Logan [13] showed that the deep drawing analyses shown below can be solved effectively with explicit codes like DYNA3D by applying loads slowly to minimize dynamic effects, so nearly quasi static solutions may be obtained.

This section briefly describes the implementation of the yield surfaces implemented into the LLNL public DYNA3D (1948 Hill as Model 33) and the internal W-DYNA (1979 Hosford and 1989 Barlat "TriComponent" as Model 33x). An overview of the explicit finite element approach used in DYNA3D is given elsewhere [13]. The relevance of material behavior enters heavily into the update of the internal force vector for an element $e$ :

$$
f_{n+1}^{i n s, e}=\int_{\Omega_{e}} B^{T} \sigma_{n+1} d \Omega_{e},
$$

using $B$, the "strain-velocity operator," and the updated Cauchy stress $\sigma_{n+1}$. The internal element force vector is assembled into the global force vector $f_{n+1}^{\text {int }}$ by assembling contributions from all elements. The stress tensor term and thus the global force vector in eqn. (1) depends in principle on the material constitutive equation chosen. In the public version of DYNA3D, we have implemented the 1948 Hill equation for anisotropic plastic flow:

$\bar{\sigma}^{2}=\frac{F\left(\sigma_{b}-\sigma_{c}\right)^{2}+G\left(\sigma_{c}-\sigma_{a}\right)^{2}+H\left(\sigma_{a}-\sigma_{b}\right)^{2}+D}{R+1}$

Eqn. (2) relates the effective stress to the three normal components of Cauchy stress, with the term $D$ containing the shear stress terms:

$$
D=2 L \sigma_{b c}^{2}+2 M \sigma_{c a}^{2}+2 N \sigma_{a b}^{2}
$$

The values for the constants in Eqns. (2) and (3) can be expressed in terms of the in-plane strain ratios $R$, $Q=R_{45}$, and $P=R_{90}$, with the following additional relations needed:

$$
\begin{aligned}
& F=S=R / P \\
& G=1 \\
& H=R \\
& L=\left(Q_{b c}+\frac{1}{2}\right)(R+I) \\
& M=\left(Q_{c a}+\frac{1}{2}\right)(R+S) \\
& N=\left(Q_{a b}+\frac{1}{2}\right)(1+S)
\end{aligned}
$$

In addition to being comparatively straightforward to implement, the quadratic 1948 Hill criterion permits the relatively simple calculation of the ratio, $X(\theta) / X$, of the yield stress in a direction at an angle in the plane of the sheet to the rolling direction, as well as the calculated $R$ value in that direction:

$$
\begin{gathered}
\frac{X(\theta)}{X}=\left[\frac{R+1}{2 N \mathrm{~s}^{2} \mathrm{c}^{2}+R\left(\mathrm{c}^{2}-\mathrm{s}^{2}\right)^{2}+\mathrm{c}^{4}+S \mathrm{~s}^{4}}\right]^{1 / 2} \\
R(\theta)=\frac{R+\left\{\left(2 Q_{a b}+1\right)(1+S)-S-1-4 R\right\} s^{2} \mathrm{c}^{2}}{S \mathrm{~s}^{2}+\mathrm{c}^{2}} \\
c=\cos (\theta) \\
s=\sin (\theta)
\end{gathered}
$$

Eqns. (6)-(7) are relatively easy to comprehend, but show several trends that are not usually borne out by experimental data. For example, at 45 degrees to the rolling direction, 1948 Hill predicts (if $R=P$ ):

$$
\frac{W}{X}=\frac{X\left(45^{\circ}\right)}{X}=\left[\frac{R+1}{Q_{a b}+1}\right]^{1 / a}
$$

The exponent ' $a$ ' is set to $a=2$ in eqn. (8) for 1948 Hill. At 90 degrees to the rolling direction, a similar situation exists for general values of unequal $R$ and $P$.

$$
\frac{Y}{X}=\frac{X\left(90^{\circ}\right)}{X}=\left[\frac{P(R+1)}{R(P+1)}\right]^{1 / a}
$$

Since the exponent ' $a$ ' is still $a=2$, Eqns. (8-9) show a high dependence of yield stress on orientation in the plane of the sheet. This dependence is a likely factor in overprediction of earing as in [4], as the strong material in the 45 degree direction tends to pull in to form the wall of the punch (forming a deep trough), while compressing the 0 degree and 90 degree walls (forming high ears). One yield criterion which has shown better agreement with experiment is the 1979 Hosford equation, extending 1948 Hill to a non-quadratic form with values of the exponent $a$ in the range of $a=8$ for fcc [7], and $a=6$ for bcc [14] metals:

$$
\bar{\sigma}^{a}=\frac{F\left(\sigma_{2}-\sigma_{3}\right)^{a}+G\left(\sigma_{3}-\sigma_{1}\right)^{a}+H\left(\sigma_{1}-\sigma_{2}\right)^{a}}{R+1}
$$

This equation predicts much milder dependencies of strength ratios in stress states and directions other than tension in the rolling direction. For example, in biaxial 
tension, where both, $\sigma_{a}=\sigma_{b}=B$, both 1948 Hill and 1979 Hosford predict the relatively simple value:

$$
\frac{B}{X}=\left(\frac{R+1}{S+1}\right)^{1 / a}
$$

Clearly, values of $a>2$ will give a much milder dependence often observed experimentally and thus should provide better correlation with LDR as shown in previous works. Further, if we assume the R-value orientation dependence as in eqn. (7), the orientation dependence of uniaxial flow stress is much milder. This ratio is important in the flange during draw-in, and has shown greater accuracy in earing calculations compared to experimental data. However, difficulties arise in the implementation of the 1979 Hosford criterion for cases in other than principal stress/strain space. This stems from the lack of shear terms in the criterion. Eqn. (10) must remain in principal stress space to be used without spurious results and non-convexity problems. To do so, we must make a key approximation in updating the Cauchy stress tensor (expressed as a vector of six) when using eqn. (10). The key necessity is a rotation to the principal stress coordinate space. This does not normally coincide with either the material (rolling and transverse direction) coordinate system, nor with the axes of principal strain. We assume that the axes of principal stress and strain approximately coincide, although for planar isotropy we know that they nomally will not. However, this assumption, which leads us to ignore cross-terms in the constitutive matrix, is believed to lead only to small errors for the degree of anisotropy observed in most sheet metals. This is the assumption we will use below to update the stresses for the 1979 Hosford criterion.

In order to circumvent the stress space limitation of the 1979 Hosford criterion, Barlat and Lian [9] introduced a criterion which offers all the advantages of the 1979 Hosford for the case of normal anisotropy $(\Delta R=0)$ but permits the introduction of a coupled shear term while retaining convexity of the yield surface and coordinate system invariance. This criterion is expressed below as in [8], except that we retain the use of 'a' as the yield criteria exponent since many of our DYNA implementations refer to ' $m$ ' as a strain-rate exponent. Note also that to avoid confusion we have expressed the coefficient (2-c) explicitly:

$$
\begin{aligned}
& \bar{\sigma}^{a}=\frac{(2-c)\left\{\left|K_{1}+K_{2}\right|^{a}+\left|K_{1}-K_{2}\right|^{a}\right\}+c\left|2 K_{2}\right|^{a}}{2} \\
& K_{1}=\frac{\sigma_{a}^{\prime}+h \sigma_{b}^{\prime}}{2} \\
& K_{2}=\sqrt{\left(\frac{\dot{\sigma_{a}}-h \sigma_{b}^{\prime}}{2}\right)^{2}+p^{2} \sigma_{a b}^{2}} \\
& \sigma_{a}^{\prime}=\sigma_{a}-\sigma_{c} \\
& \dot{\sigma_{b}}=\sigma_{b}-\sigma_{c}
\end{aligned}
$$

The Cauchy stresses must be defined to allow for a third (normal) stress, even though the implementation here is for the shell element in DYNA. This is because the plane-stress material routine is iterative so that even though the normal stress vanishes at convergence, we must recognize its presence during the iterations. Parameters $c, h$, and $p^{*}$ may be defined in the current notation as follows:

$$
\begin{aligned}
& c=2 \sqrt{\left(\frac{R}{1+R}\right)\left(\frac{P}{1+P}\right)} \\
& h=\sqrt{\frac{R(1+P)}{P(1+R)}} \\
& p^{*}=\sqrt{\frac{\left(2 Q_{a b}+1\right)(1+S)}{(1+R)(2+c)}}
\end{aligned}
$$

The value of $p$ is needed for the shear term in eqn (12). In the case of $a=2$, we have $p=p^{*}$. However, this is also the case where the criterion reduces identically to 1948 Hill and is thus of interest only for verification. In general, the value of $p^{*}$ must be found iteratively as described by Barlat and Lian in [9]. However, this has not been found to be a drawback for the case considered thus far where these coefficients are constant.

Given either of the choices of yield surface, the next step in implementation involves the stress update. For the isotropic case, this is conveniently done using the radial retum method as discussed by Krieg and Key [15]. However, this method cannot be used directly for anisotropic plasticity. Thus, the incremental method described by Bathe [16] and others is used in the DYNA implementation. Due to the small strain increments typical in an explicit dynamics code, this again is not a major drawback in computation speed. The incremental stress update involves calculation of contact stresses and updated elastoplastic stresses as follows:

$$
\begin{aligned}
& \sigma_{i}^{c}=\sigma_{i}^{n}+C_{i j}^{e} d \varepsilon_{j}^{e} \\
& \sigma_{i}^{N}=\sigma_{i}^{c}+C_{i j}^{e p}\left(d \varepsilon_{j}-d \varepsilon_{j}^{e}\right)
\end{aligned}
$$

Here, $d \varepsilon_{j}^{e}$ are the elastic portions of the strain increment, and $C_{i j}^{e}$ is the elastic constitutive matrix. To obtain the updated stresses, we proceed further by applying the remainder of the strain increment $\left(d \varepsilon_{j}-d \varepsilon_{j}^{e}\right)$ using the elastoplastic matrix $C_{i j}^{e p}$ :

$$
C_{i j}^{e p}=C_{i j}^{e}-\frac{C_{i j}^{e} q_{j}\left(C_{i j}^{e} q_{j}\right)^{T}}{p_{i} q_{i}+q_{i}^{T} C_{i j}^{e} q_{j}}
$$

The yield surface $F$ directly affects the calculation of the matrix $C_{i j}^{e p}$, since

$$
q_{i}=\frac{d F}{d \sigma_{i}}
$$




$$
p_{i}=-\frac{d F}{d \varepsilon_{i}}
$$

In the following sections we will demonstrate the effect of the chosen yield surface (Eqn. (2), (10), or (12)) on the extent of earing in cupping, and on the choice of optimal blank shape for minimization of material usage during drawing of square and cylindrical cups.

\section{EARING DEVELOPMENT AND FINITE-ELEMENT PREDICTIONS}

To demonstrate the correlation the different yield criteria in DYNA with earing observed experimentally, we compare the data obtained by Wilson and Butler [1] with that obtained using simulations with either $a=2$ in eqn. (2) (1948 Hill), $a=8$ in eqn. (10) (1979 Hosford), or $a=8$ in eqn. (12) (1989 Barlat). Numerous runs with $R=$ $P$ were made as outlined in detail previously [4] for the first two of the three criteria outlined here, using a $100 \mathrm{~mm}$ punch and $200 \mathrm{~mm}$ diameter blank. To explore what we might expect to find for a moment, we consider two sets of values for $R, Q_{a b}$, and $P$ that typify the magnitudes commonly observed in AK and IF steels. In fact, the dependencies are not far from those observed in warm rolled and annealed D38 uranium [11], depending on whether unidirectional or cross-rolling was employed:

TABLE 1. Plastic material properties used in the DYNA analyses in the current work.

\begin{tabular}{|l|r|r|r|r|r|}
\hline Matenal & $K($ MPa) & $n$ & $A$ & $Q_{a b}$ & $P$ \\
\hline AK & 560 & .24 & 2.500 & 1.500 & 2.500 \\
\hline IF & - & - & 1.500 & 2.000 & 2.500 \\
\hline SS304 & 1514 & .43 & 1.025 & 1.025 & 1.025 \\
\hline D38-iV & 1300 & .24 & 2.800 & 1.400 & 2.800 \\
\hline
\end{tabular}

The values of $K$ and $n$ refer to the equation:

$$
\sigma_{a}=K \varepsilon_{a}^{n}
$$

used in tabular form in W-DYNA's Model 33x to describe the uniaxial stress-strain behavior in the a-direction.

The values in Table 1 lead to some interesting predicted dependencies of stress on direction and stress state as shown here in Fig. 1. As noted above, the W/X ratio for the 'AK' steel is much lower for the 79 Hosford than for 48 Hill, which explains the improved earing correlation noted in [4]. The W/X ratio for the 89 Barlat is intermediate between the two. Finding the value of $W / X$ for the 89 Barlat requires the same iterative procedure used to find the value of $p$ in eqn. (12). Thus, we might anticipate earing behavior for 89 Barlat that is intermediate between the 48 Hill and 79 Hosford criteria. The $B / X$ ratio does not directly enter into the earing behavior, but will enter directly into the blank shape optimization to follow as we will need to consider thinning at the punch nose as well as earing in the flange. In this respect, the 79 Hosford and 89 Barlat criteria predict the same value which is much lower than 48 Hill as explained above. The third ratio explored in Fig. 1 is the $Y / X$ ratio or ratio of yields in the rolling and transverse directions. This ratio was calculated for the 'IF steel using rounded $R$-values typical of that material and perhaps unidirectionally rolled and annealed D38 as well. In contrast to the $\mathrm{B} / \mathrm{X}$ ratio, the 89 Barlat (Tri-
Component) yield surface here shows a $Y / X$ ratio identical to 48 Hill and not 79 Hosford. Thus, predicted behavior in the flange for IF-like materials where $R$ and $P$ are unequal may show quite a different comparison and this issue is reserved for future study.

48 Hill $a=2$

(3) 79 Hosford $a=8$

D 89 Barlat $a=8$

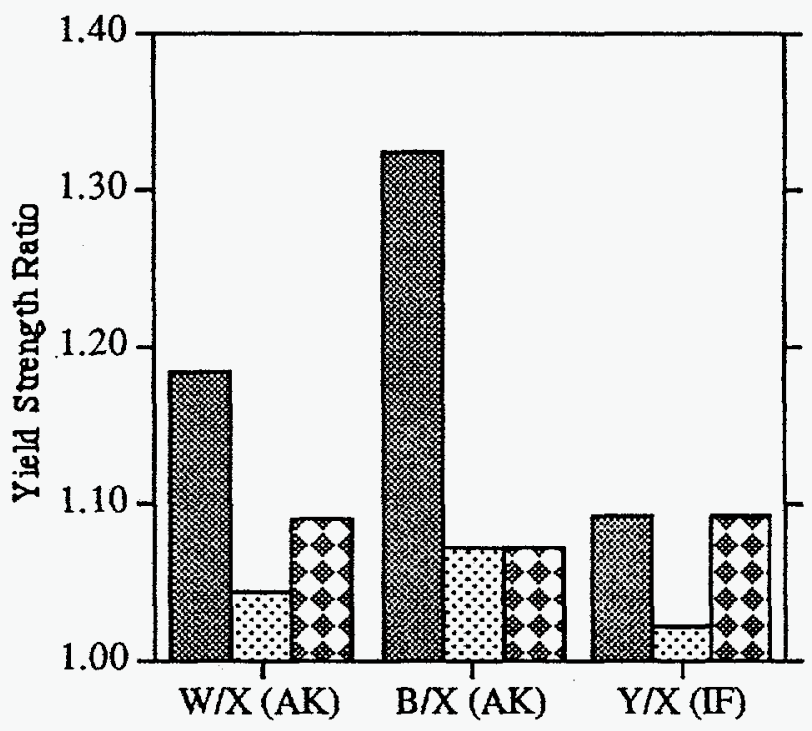

Direction and (Material)

Fig. 1. Comparison of yield strength ratios as predicted using the three plane-stress yield criteria in this work for materials where $R=P(A K)$ and $R>P$ (IF).

In light of the strength ratio $(W / X)$ observed in Fig. 1, earing calculations performed in [4] were repeated using the 89 Barlat criterion with exponent $a=8$. These results are plotted as $\Delta h / h$ vs. $\Delta R / R$ in Fig. 2 , along with the original data from Wilson and Butler. As noted in [4], it is clear that the use of $a=2$ (identically 1948 Hill) over predicts earing by about a factor of two. In contrast, the use of $a=8$ in the 79 Hosford equation matches almost exactly the observed earing data.

As might be expected from the trend of $(W / X)$ implied in Fig. 1, the 89 Barlat predictions are intermediate between 48 Hill and 79 Hosford, the latter of which matches best the experimental data. This situation may change for cases where $R$ and $P$ are unequal, or where yet another yield surface, perhaps the 6component surface of Barlat et al. [17] is used. 


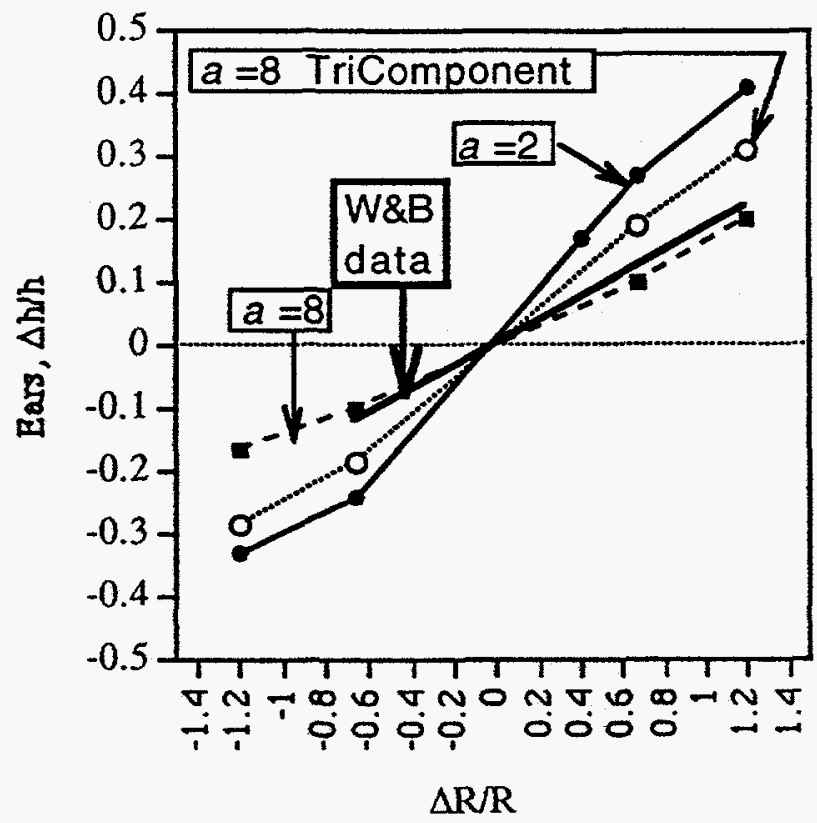

Fig. 2. Plot of earing ( $\Delta h / \mathrm{h}$ vs. $\Delta R / R)$ including Wilson and Butler data compared to DYNA results with $a=2$ (48 Hill), $a=8$ ( 79 Hosford) and $a=8$ (89 Barlat).

\section{SQUARE-CUP DRAWING: BLANK SHAPE EFFECT}

Given an understanding of the capabilities of the three yield criteria above regarding earing, we next desired to explore their influence in determining an optimal blank shape in square cup drawing. This was done previously for the case of isotropic material by Kobayashi et al. [10] for the stamping of a small $(40 \mathrm{~mm} x$ $40 \mathrm{~mm}$ punch) square cup. Blank shapes of a circle, octagon, and square (all having the same initial volume) were used with a 304 stainless steel as characterized in Table 1. The experimental punch force vs. punch depth behavior is reproduced in Fig. 3 for those geometries and compared to analyses with DYNA pertormed in this work. The bold lines in Fig. 3 represent the current DYNA results, while the lighter lines (coded the same way) represent the finite-element (FEM) results as reported in [10]. It was noted in [10] that their FEM punch forces tended to be lower than experimental, but they did reproduce the trend of punch force as a function of blank shape. These same trends are shown in the current DYNA simulations, with improved correlation to experiment. However, it is not clear whether the improved agreement in this work is serendipitous or due to improved accuracy in the code or shell element formulation. As Kobayashi et al. point out the discrepancy may be due to an increasing friction coefficient during the stamping process. This possibility was neglected in both [10] and in the current work. However, this work did use the Selective Velocity Enhancement (SVE) technique for quasi-static simulation with the explicit DYNA code [13], and this can result in increased punch forces. Several values of SVE were tried and this does not appear to be the case, so the reason for improved punch agreement is not clear, although it is certainly desirable.

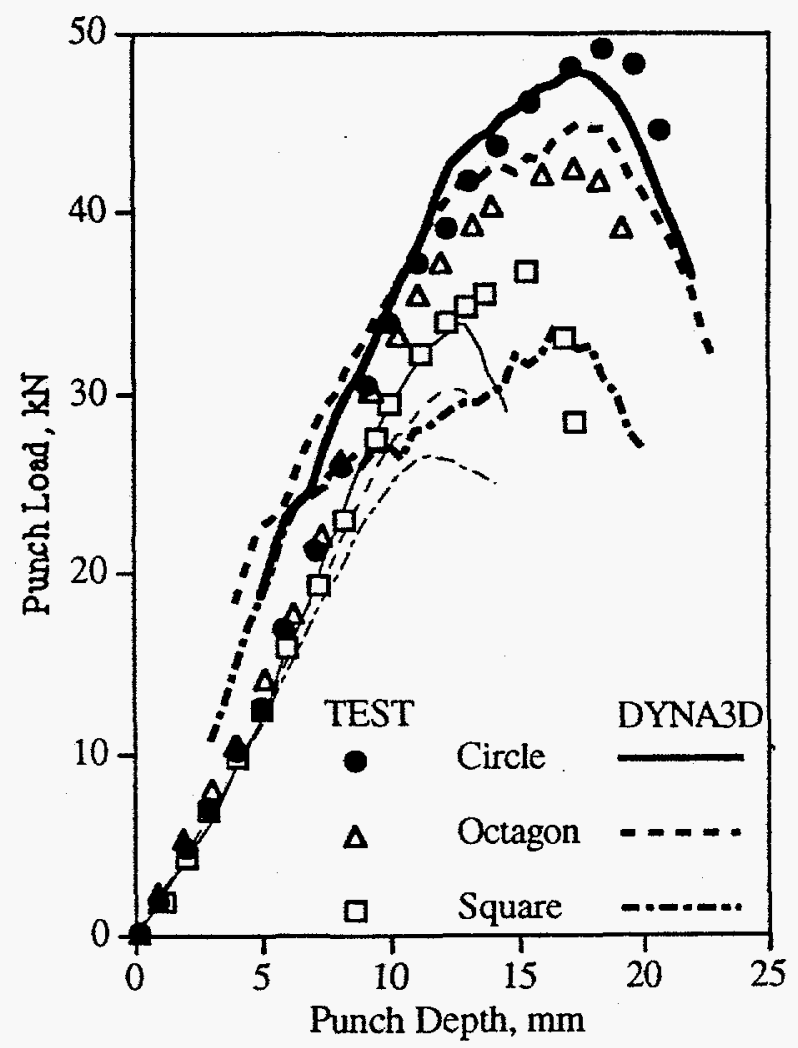

Fig. 3. Comparison of punch force vs. displacement for the experiments as reported in [10] vs. the current DYNA3D results (bold lines) and the FEM results in [10] (fine lines) as a function of blank shape.

TABLE 2. Comparison of peak normal strains as calculated in the current work and in [10] for the experiments in [10], transverse cut to mid-side of box.

\begin{tabular}{|c|c|c|c|c|}
\hline & \multicolumn{2}{|c|}{$\begin{array}{l}\text { Peak Thinning } \\
\text { Strain \% (Punch) }\end{array}$} & \multicolumn{2}{|c|}{$\begin{array}{l}\text { Peak Thickening } \\
\text { Strain \% (Flange) }\end{array}$} \\
\hline Blank & Reflio] & DYNÁ & Refl70] & DYINA \\
\hline Circle & 3 & 4 & $\overrightarrow{7}$ & $\overline{6}$ \\
\hline Octagon & 3 & 4 & $\sqrt{3}$ & 12 \\
\hline Square & 3 & 4 & 12 & 12 \\
\hline
\end{tabular}

TABLE 3. Comparison of peak normal strains as calculated in the current work and in [10] for the experiments in [10], diagonal cut to comer of box.

\begin{tabular}{|c|c|c|c|c|}
\hline & \multicolumn{2}{|c|}{$\begin{array}{l}\text { Peak Thinning } \\
\text { Strain \% (Punch) }\end{array}$} & \multicolumn{2}{|c|}{$\begin{array}{l}\text { Peak Thickening } \\
\text { Strain \% (Flange) }\end{array}$} \\
\hline Blank & Rellio] & DYNA & Refl[0] & DYINA \\
\hline Circle & $\overline{13}$ & 15 & 16 & 13 \\
\hline Octagon & 76 & 75 & 9 & 9 \\
\hline Square & 77 & 15 & 9 & 5 \\
\hline
\end{tabular}


In contrast to the punch forces, there is good agreement between the peak normal strains (thinning at the punch nose and thickening in the flange) between the current DYNA runs and the FEM of [10]. This is shown in Table 2 for a transverse cut to the mid-side of the box, and in Table 3 for a diagonal cut to the box comer, showing the peak thinning under the punch nose comer. The slight increase in thinning and decrease in thickening are indicative of a slight SVE affect in the current explicit DYNA analyses and thus might partially account for the higher punch forces.

\section{SQUARE-CUP DRAWING: ANISOTROPY EFFECTS}

Given the good agreement between the current DYNA analyses and the experimental and FEM data reported in [10], we chose to explore the influence on the choice of anisotropy model on the optimum shape of blank for the forming of a square cup such as the one here. The optimized blank shape was chosen to retain the original blank volume as in the above isotropic study. Criterion for success was chosen to maximize the height of the square cup formed, multiplied by the thinnest resulting portion of the formed box, with the implicit assumption of some minimum wall thickness in the final product that must be retained; thus the parameter "adjusted draw depth" reported in the figures below refers to the minimum wall height (troughs of the ears) multiplied by the thickness (relative to the original) at the thinnest point in the final part (usually the punch nose comer).

Some early runs were made using octagon shaped blanks with various amounts trimmed from the comers, but it was found that secondary troughs were the usual result, and that there was no simple way to characterize the transition in blank shape to the circle, even though that blank shape proved to be near (but not at) the optimum for this case of forming with no blankholder or drawbead restraints as in [10]. Thus, the transition from a round to square blank was made by adding a linear section to the blank but retaining rounded corners, which then decreased in size to retain the original volume of the $80 \mathrm{~mm}$ diameter circular blank. The blank shape could then be expressed as a Percentage Toward a Square (PTS) blank, where the rounded comers disappeared completely at $100 \%$ for the square blank. Note that the optimum blank shape was in some cases a negative percent, where $-100 \%$ would represent a square blank rotated $\mathbf{4 5}$ degrees from square placement over the square die. The qualitative results achieved are shown below for the three basic blank shapes in Figs. 4-6, for the assumption of the 48 Hill criterion and $\triangle R>0$ (i.e. rolling direction along the sides of the box). Fig. 4 shows the box shape formed from a square blank (PTS $=100$ ) and the large ears as expected at the box comers. Fig. 5 shows the opposite case, with PTS $=-40$ (rotated blank and rounded comers) showing ears on the mid sides of the box. Fig. 6 shows the circular blank which tumed out to be the optimal blank shape for this case of yield criterion and material rotation. The upper mesh in each case shows the initial blank shape, with shading representing the amount of thickening during the drawing operation. The unshaded areas indicate a normal strain of -0.08 or below, while the darkest areas indicate a normal strain of +0.08 or above in the flange.
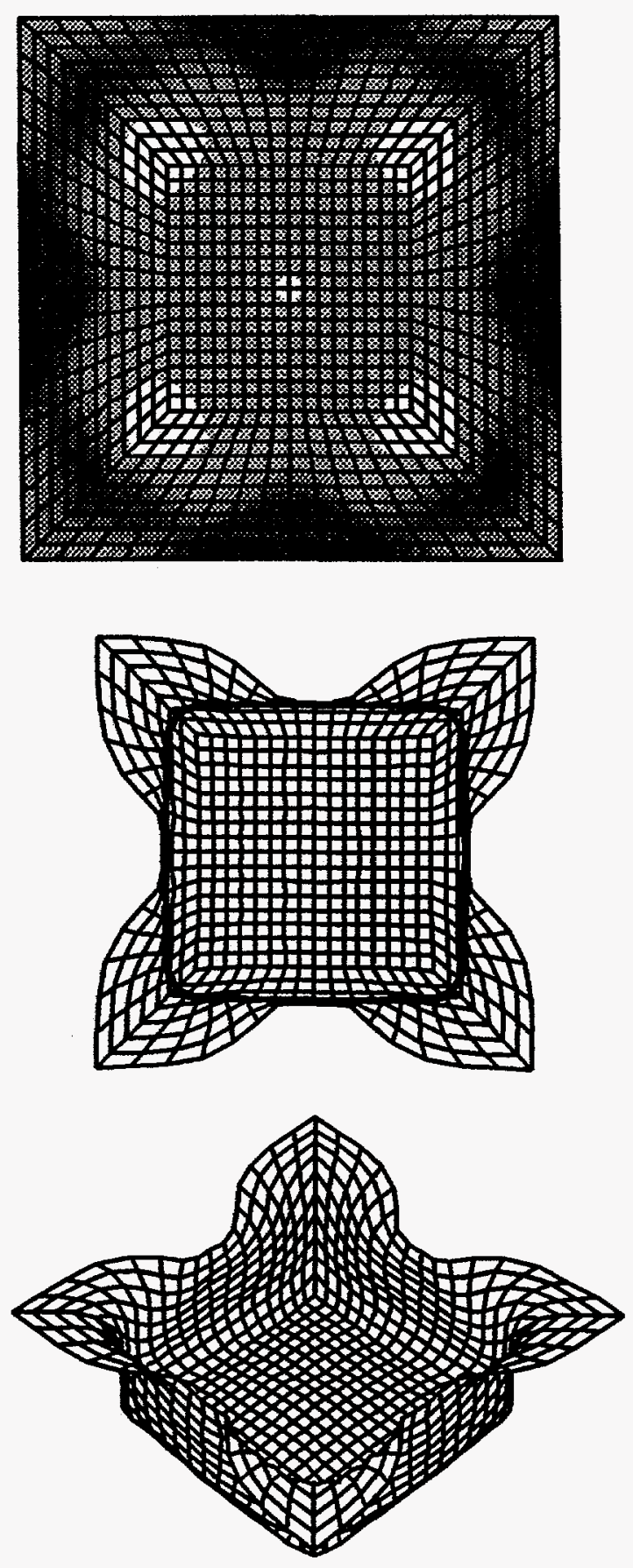

Fig. 4. Forming of square-cup box using square (PTS $=100$ ) blank with placement so $\Delta R>0$ with $48 \mathrm{Hill}$. 

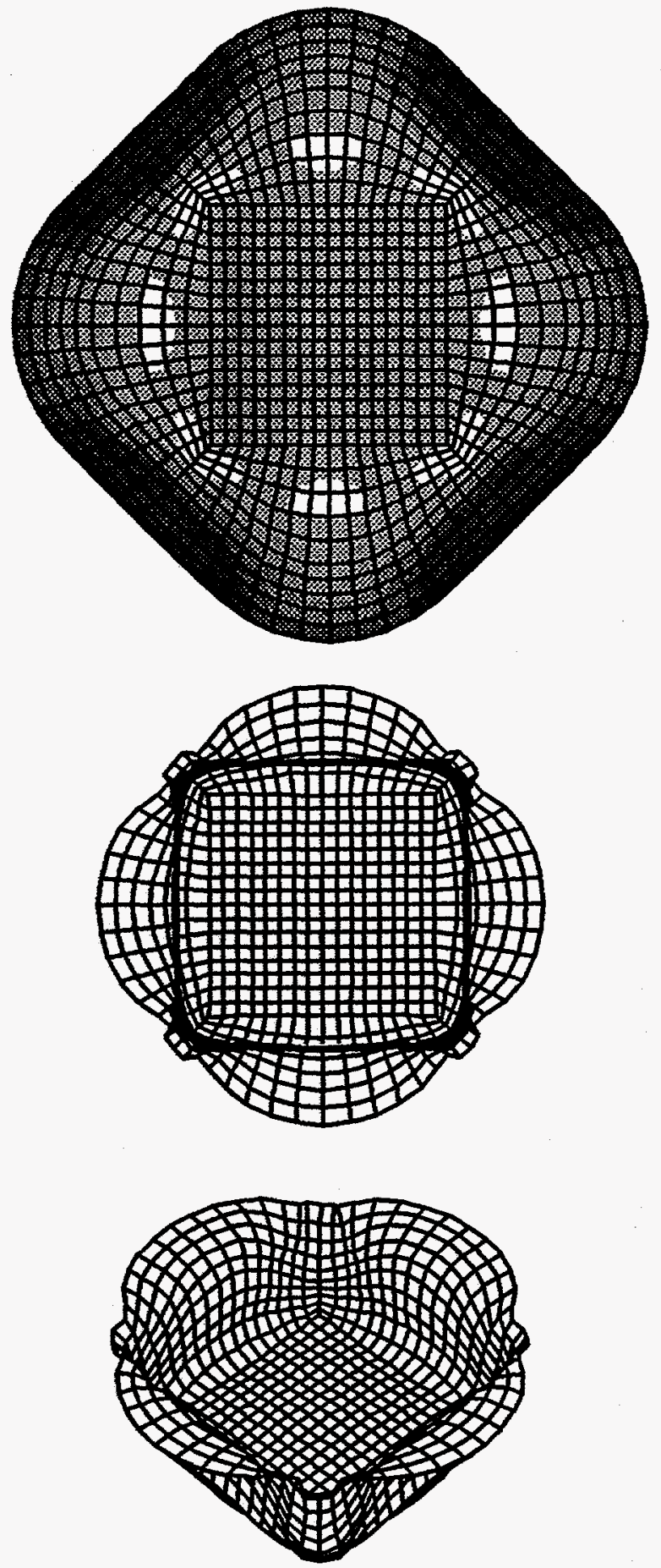

Fig. 5. Forming of square-cup box using rounded and rotated (PTS $=-40$ ) blank with placement so the adirection is along the blank mid-side, 48 Hill criterion used.
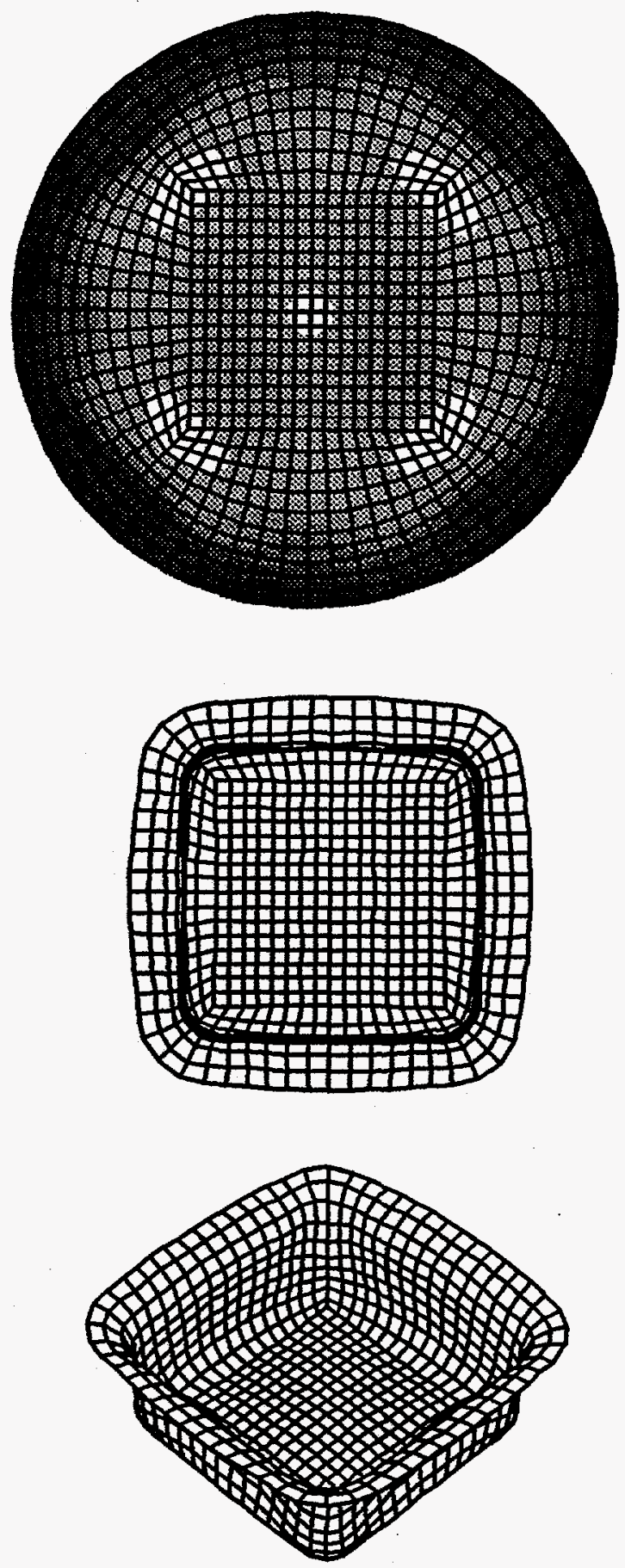

Fig. 6. Forming of square-cup box using circular (PTS $=0$ ) blank with placement so the a-direction is along the blank mid-side of the die, 48 Hill criterion used. 
With the general trends observed in Figs. 4-6 in mind, we then chose to determine the optimum blank shape (PTS value) as determined by the assumption of anisotropy and the yield criterion chosen to represent this anisotropy. As a baseline, the $48 \mathrm{Hill}$ criterion was used with assumptions of normal anisotropy only $(\triangle A=0)$, planar anisotropy with the a-direction along the mid-side of the blank $(\triangle B>0)$, planar anisotropy with the a-direction toward the comer of the blank $(\Delta R<0)$, and finally the isotropic (von Mises for the exponent $a=2$ ) case for comparison. In all cases as shown in Fig. $7 a$, the optimum blank shape occurs at PTS $=0$ or $\mathrm{PTS}<0$. For the $\triangle A=0$ case, we obtain $P T S=-16$ with an adjusted draw depth $A D D=20.12 \mathrm{~mm}$. The best result occurs with a $\triangle R>0$ placement and $P T S=0$ (circular blank), with an $\mathrm{ADD}=20.24 \mathrm{~mm}$. The case for $\Delta R<0$ shows an optimum PTS $=-20$ with $A D D=20.06 \mathrm{~mm}$. It is interesting to note that (as expected), blank placement so that $\triangle R>0$ shows a higher $A D D$ and changes the PTS, but that placement so $\triangle R<0$ does not seem to adversely affect the results. This is consistent with an overall trend observed for this square-cup drawing process in that the choice of yield criterion and use of normal anisotropy (compared to $R=1$ ) had a much greater affect than the effect of $\Delta R$ value and placement. The most noticeable reduction in ADD occurred for the von Mises assumption, with an ADD of only $18.15 \mathrm{~mm}$ at PTS $=0$, thus showing a difference of almost $12 \%$ in ADD depending on the anisotropy assumption.

Use of the 79 Hosford criterion with exponent $a=8$ showed (Fig. $7 \mathrm{~b}$ ) the expected results on the trends of PTS and ADD. All of the peak values of ADD now occur at $P T S=-20$, and the values of $A D D$ are much closer, with $A D D=19.67 \mathrm{~mm}$ for $\triangle A=0$ and $A D D=18.91 \mathrm{~mm}$ for $R=1$ (close to Tresca criterion) representing the extreme differences of only $4 \%$ in ADD. The 89 Barlat criterion (Fig. 7C) showed a similar tightening of the band of ADD, with the notable difference being the change in place of the $\triangle A>0$ and $\Delta R<0$ cases. The maximum $A D D=19.87 \mathrm{~mm}$ at $P T S=-20$ is now due to the $\Delta R<0$ condition, with the minimum again due to an $R=1$ assumption with $A D D=18.82 \mathrm{~mm}$ at PTS $=-20$. Again, the difference in ADD is only $5.6 \%$ over the range of assumed anisotropy.

Of equal importance in the blank shape issue is the effect of the chosen yield criterion for any given level of anisotropy. These comparisons are made in Figs. 8a$8 \mathrm{~d}$ by rearranging the data discussed in Fig. $7 \mathrm{a}-7 \mathrm{c}$ and grouping for a given degree of anisotropy. Thus, although the plots in Figs. $8 a-8 d$ represent approximations to the anisotropy of a given material, they might well represent those of separate but actual materials (for example, the $R=1$ condition in Fig. $8 \mathrm{~d}$ approximates the isotropy of the 304 stainless steel used in [10]. Use of the 48 Hill criterion $(a=2)$ shows the greatest differences compared to the two criteria with $a=8$, especially for the cases where $\Delta R>0$ and $R=1$. Predictions for the peak value of $A D D$ again are interchanged for the $\triangle A>0$ and $\triangle R<0$ cases depending on the use of the 79 Hosford or 89 Barlat criteria.

Overall, for this case of square-cup forming, the use of any of the three criteria will give a reasonable result, given that (at least) normal anisotropy is considered. The tendencies of $48 \mathrm{Hill}$ to overpredict earing and LDR do not adversely affect either the choice of blank shape (PTS) or the resulting adjusted blank height (ADD) for the forming conditions here.
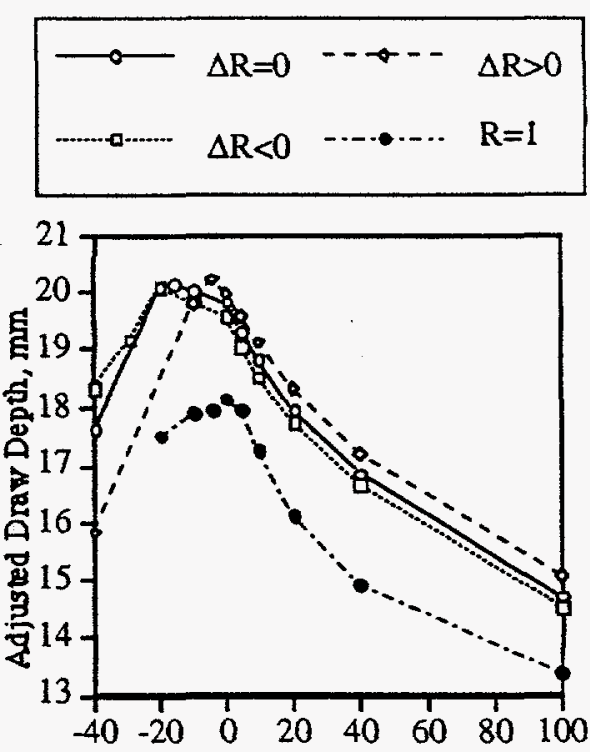

Percent Toward Square Blank
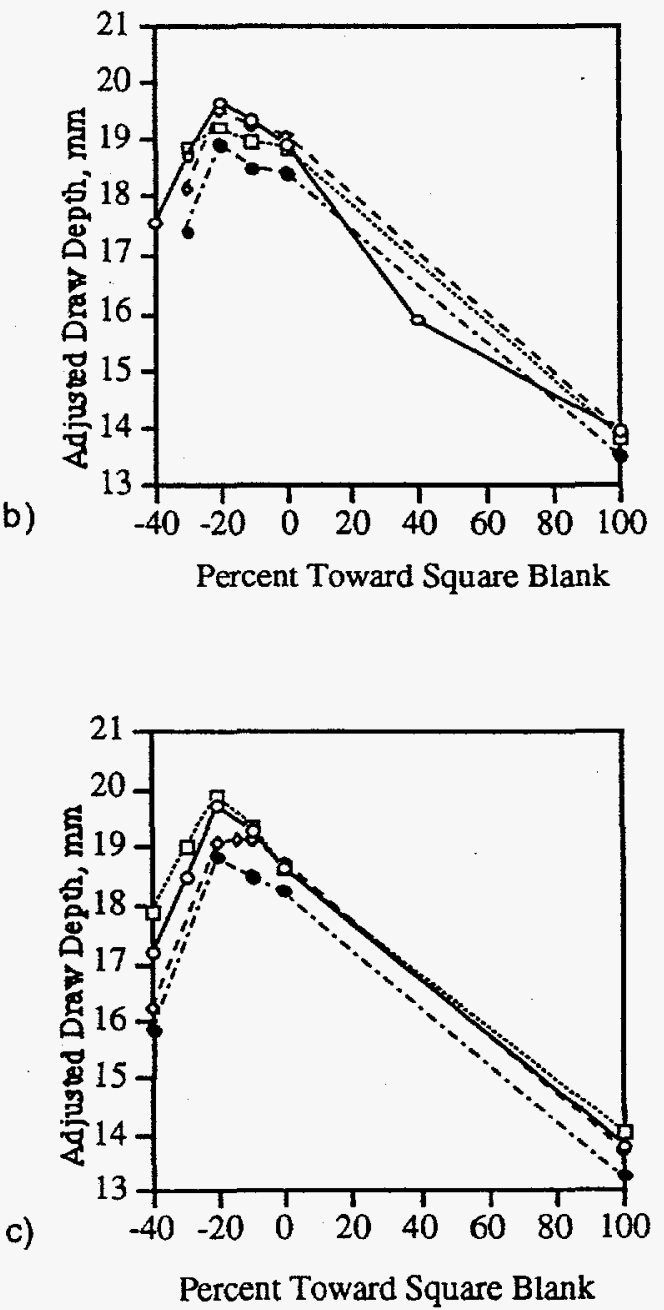

Fig. 7. Dependence of ADD on PTS for anisotropy assumptions (top) and yield criterion chosen: (a) 48 Hill, (b) 79 Hosford, (c) 89 Barlat. 


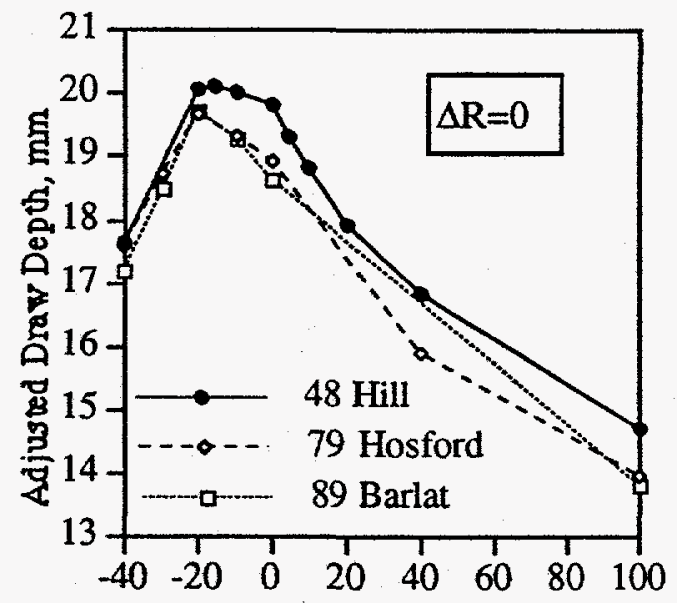

Percent Toward Square Blank

b)

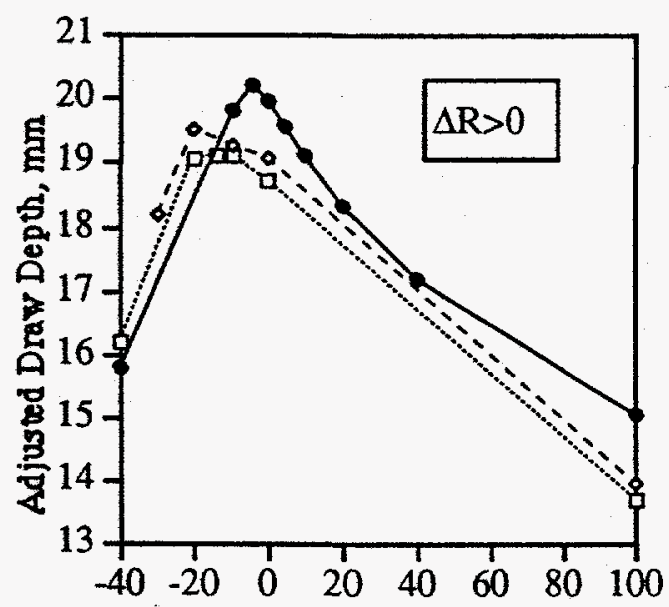

Percent Toward Square Blank

Fig. 8a-b. Plot of depth ADD vs. blank shape PTS for the cases (a) $\Delta R=0$ and (b) $\Delta R>0$. Use of the three choices of yield criteria are compared. Results are similar for (a) $\Delta R=0$, with 48 Hill showing a more rounded PTS dependence. For $\triangle R>0$, the optimum PTS and $A D D$ achieved differ notably with 48 Hill compared to the nonquadratics.

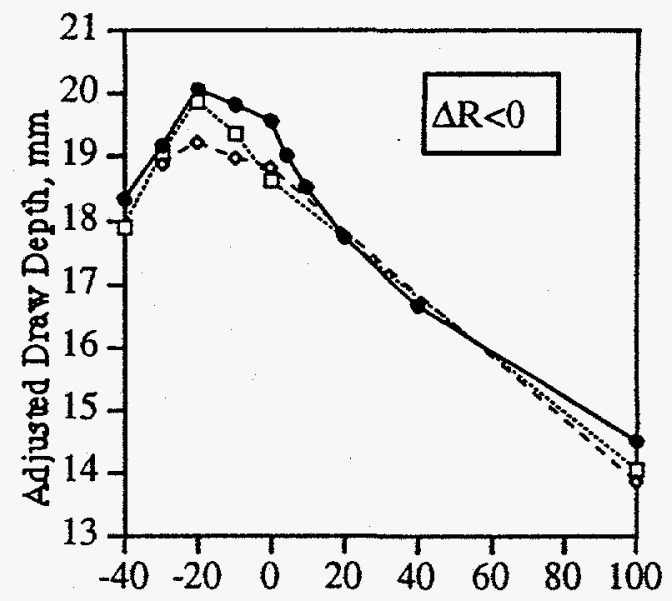

Percent Toward Square Blank

d)

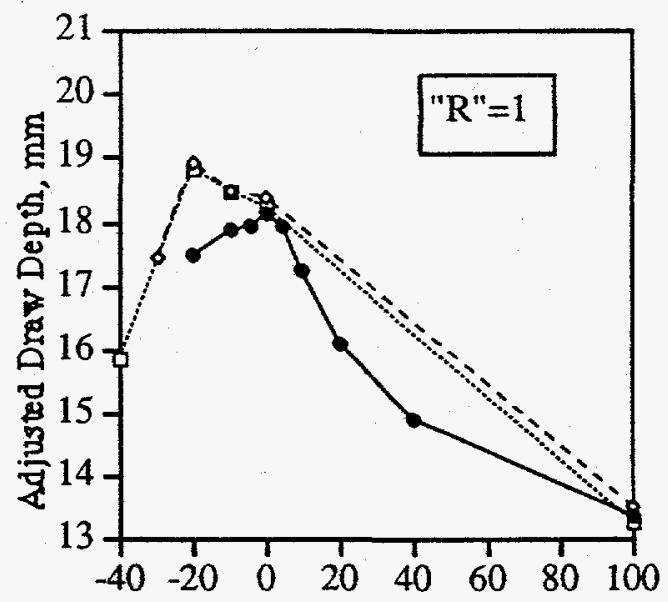

Percent Toward Square Blank

Fig. 8c-d. Plot of depth ADD vs. blank shape PTS for the cases (c) $\Delta R<0$ and (d) $R=1$. Use of the three choices of yield criteria are compared. Results for $\Delta R<0$ show a reduced ADD predicted by the 79 Hosford criterion, while 89 Barlat is close to 48 Hill despite the different exponents. For the $R=1$ isotropic case (d), 79 Hosford and 89 Barlat are nearly coincident while 48 Hill shows a lower ADD at different PTS; expected due to the high 48 Hill dependence of LDR on normal anisotropy. 
a)
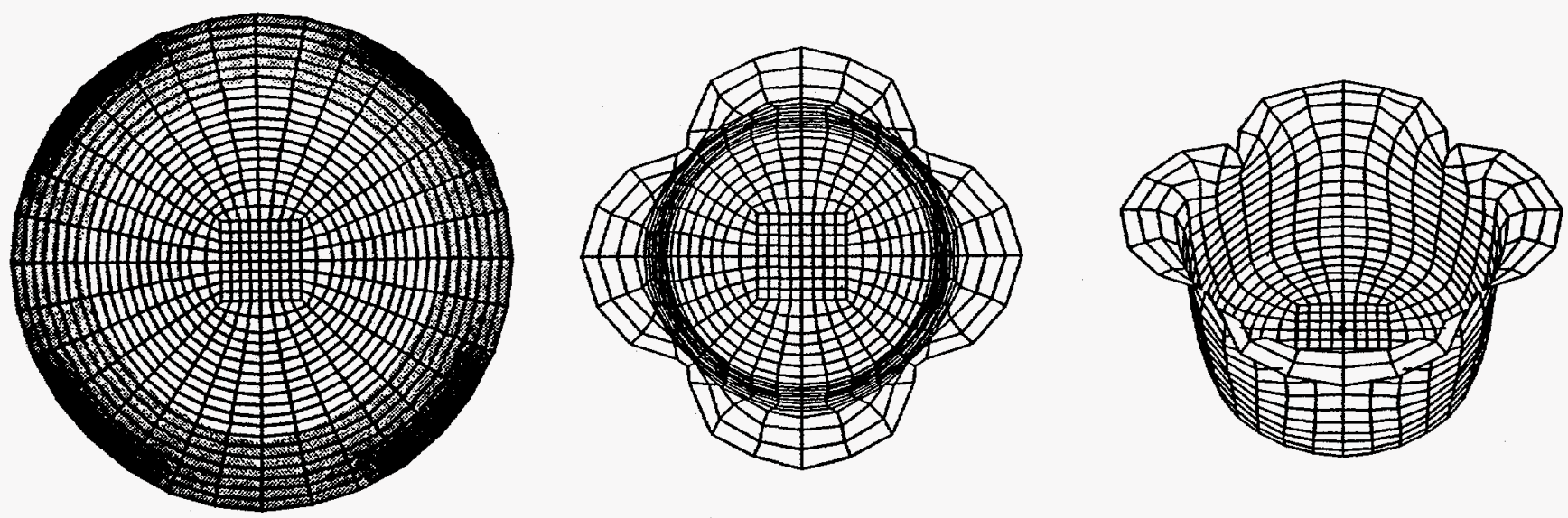

b)
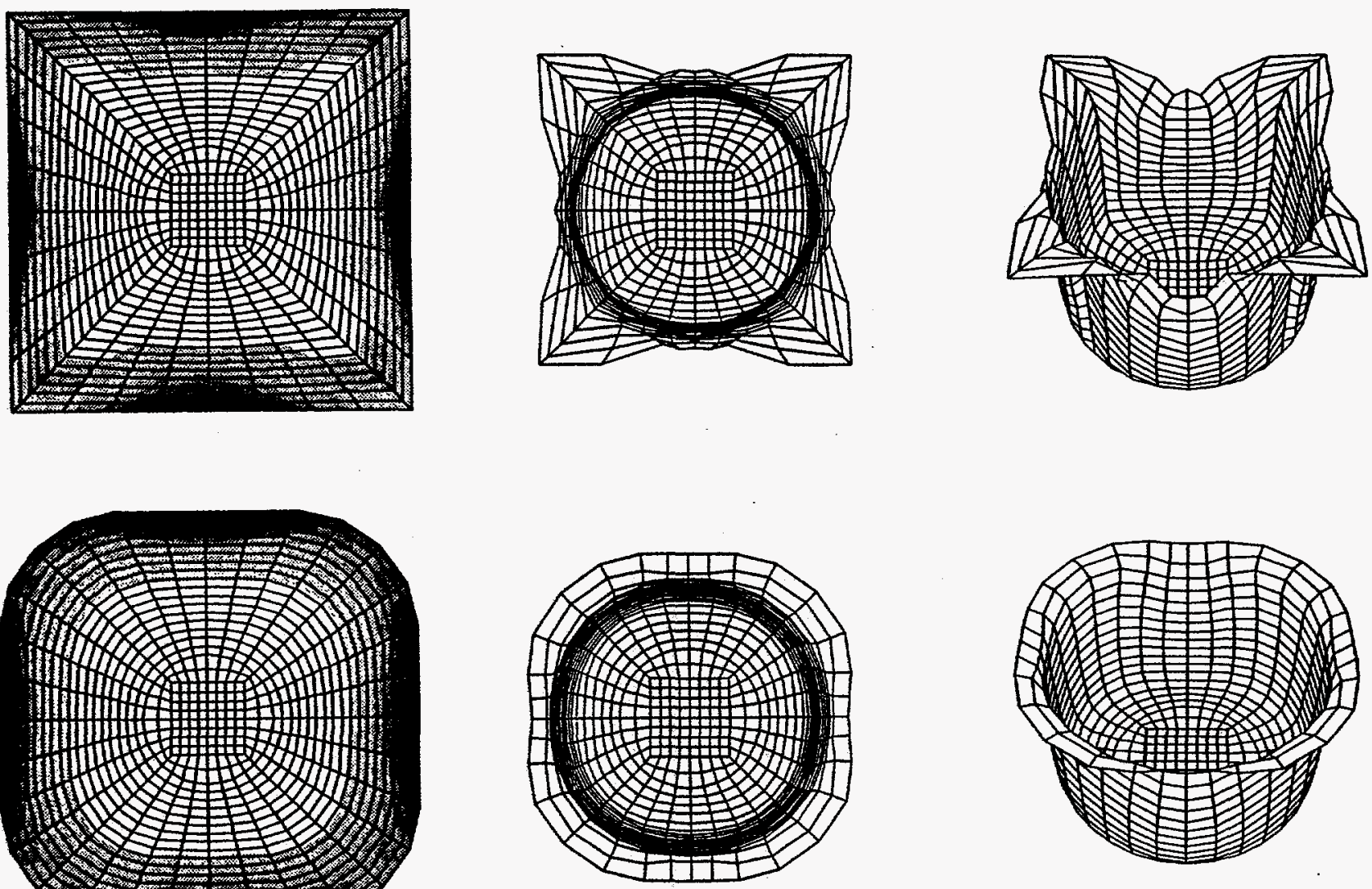

c)

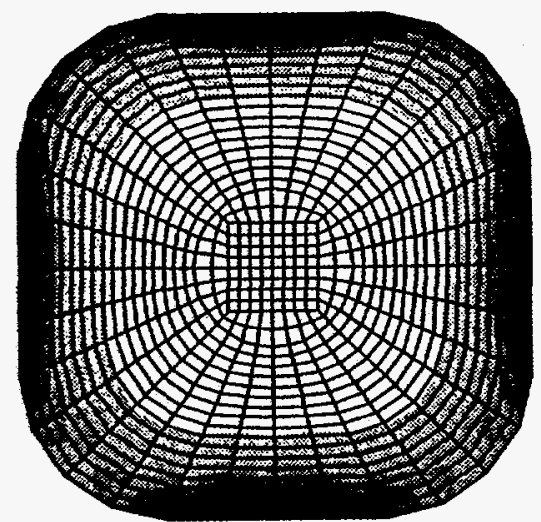

Fig. 9a-c. Sequence of blank shapes in optimization of cylindrical cupping operation in the warm forming of D38 uranium with $\triangle A>0$ (a-direction along mid-sides) and use of 48 Hill yield criterion. (a) Initial blank with PTS $=0$ (round blank). Ears form as expected from earing studies in Fig. 2. (b) Square blank (PTS=100) shows ears at the diagonals; blank shape has overcome effect of $\triangle A>0$. (c) Blank with PTS=40, near optimum for assumption of 48 Hill.

\section{ANISOTROPIC D38 URANIUM CUP DRAWING}

In order to characterize the drawability of D38, we chose to model the forming process and geometry of the "MTS" type Formability Tester (Materials Testing Systems, Minneapolis, MN) "standard" (100mm die) cupping facility as shown in Fig. 9a-c. It has been shown by Hughes et al. [11] that optimal processing of the unalloyed D38 uranium alloy can result in planar anisotropy with the values of $R, Q_{a b}$, and $P$ as shown in Table 1 above as reported in [11] for the case of $20 \%$ work followed by an anneal. In a similar manner to the above square-cup study, we choose here to determine the optimal blank shape (PTS) for this cylindrical cupping operation with $\Delta R>0$. As can be seen from the examples shown in Flg. 9a-c, the optimum appears at PTS $=40$, resulting in a nearly uniform thickening (darkened region) in the flange of the cup, although the greatest thickening is still in the diagonal direction where $Q_{a b}=1.4$ as opposed to $R=P=2.8$ in the mid-side direction.

It is apparent even qualitatively from Fig. 9 that there is a large change in blank shape needed to counter the strong effect of planar anisotropy present, since in the absence of planar anisotropy a circular blank would clearly be the correct choice. A detailed examination of 
the ADD achieved from a circular blank can be made as a function of anisotropy and yield criterion assumption, and the results of this are shown in the bar graphs of Fig. 10a-b. For this cupping operation, we use a $100 \mathrm{~mm}$ punch with a $200 \mathrm{~mm}$ blank for a draw ratio of 2.0 . Thus, in addition to the earing effect, the adjusted draw depth ADD will be affected by the LDR that would be predicted due to each of the yield criteria. This is shown immediately in Flg. 10a even for the $\triangle R=0$ case, where 48 Hill predicts a much higher ADD due to the fact that an average value of $\bar{R}=2.1$ is used. In spite of this, the highest ADD is predicted by the 79 Hosford criterion for $\Delta R>0$, as the 48 Hill criterion predicts a much higher earing percent which is detrimental with the use of a round blank. Note though that the 89 Barlat predicts nearly as strong a penalty for the $\Delta R>0$ case. For the isotropic case with $\bar{R}=1.0,48$ Hill again predicts a severe reduction in ADD, compared to $\bar{R}=2.1$, while both 79 Hosford and 89 Barlat show a much milder reduction. Note that in fact, for the two cases in Fig. 10a with $\Delta R=0$, the 79 Hosford and 89 Barlat criteria should in fact give identical results. We must remember the subtle difference in implementation, however, and the approximation of coincident principal stress and strain for the 79 Hosford case. This may cause some artificial rounding of the corners of the yield surface, effectively lowering the a-value exponent in that sense. This effect, though small, is more noticeable for the $\bar{R}=1.0$ case, which is closer to the LDR and thus experiences more thinning. This may make the 89 Barlat implementation slightly more accurate for cases where $\Delta R=0$ and we are close to the material's forming limit.

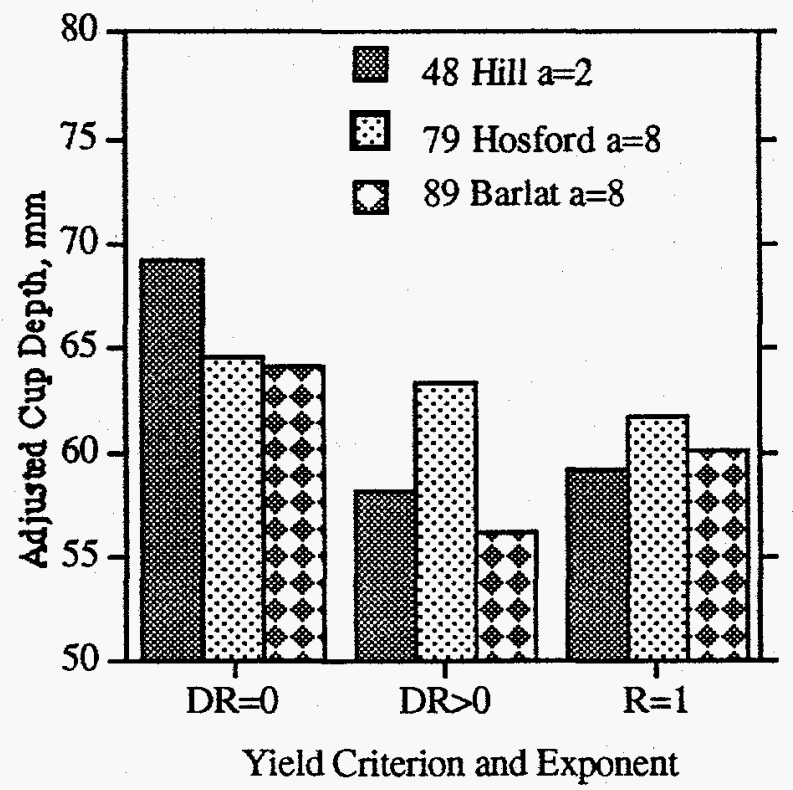

Fig. 10a. Dependence of adjusted draw depth (ADD) on assumptions of anisotropy and yield criterion; effect of yield criterion compared.

Fig. 10b rearranges the data above in a way to show the sensitivity of each criterion to the assumed anisotropy. As expected, 48 Hill shows a strong reduction in $\mathrm{ADD}$ due to either the $\Delta R>0$ or $\bar{R}=1.0$ assumption, while the 79 Hosford is rather insensitive to either. The 89 Barlat is most sensitive to the $\Delta R>0$ condition, consistent with the observations above.

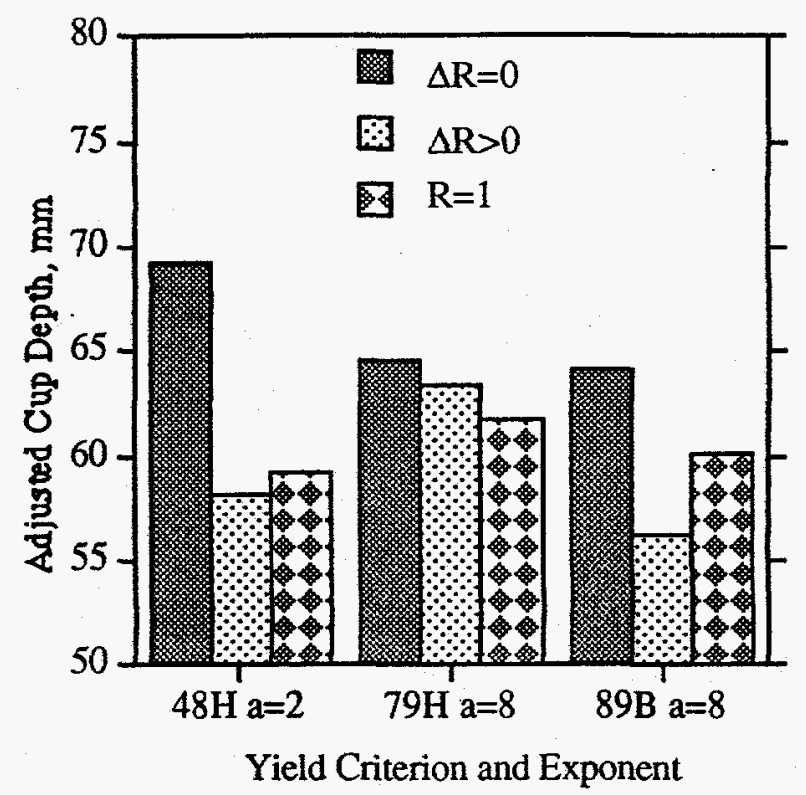

Fig. 10b. Dependence of adjusted draw depth (ADD) on assumptions of anisotropy and yield criterion; effect of anisotropy compared.

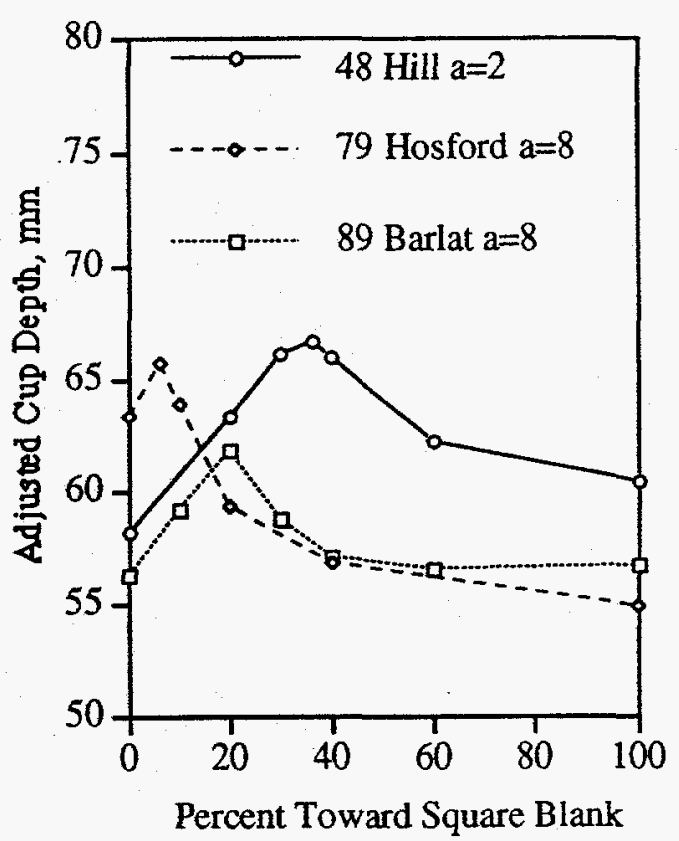

Fig. 11. Determination of optimum blank shape (PTS) for the forming of cylindrical cups from D38 uranium with planar anisotropy; effect of yield criterion.

Fig. 11 shows the effect of assumed yield criterion on the blank optimization effort which is only meaningful for $\Delta R>0$; the case for $\Delta R<0$ is obviously symmetric. All of the criteria show a strong dependence of ADD on PTS as the optimum PTS is approached, 
with the 48 Hill showing PTS=36 as the greatest deviation from a circular blank. The optimum for the 79 Hosford criterion is, as expected, the closest to a circular blank at PTS $=6$. The 89 Barlat optimum is as might be expected between the two others at PTS $=20$, but with a much lower value of ADD at the optimum, due to its unique prediction of a high dependence of earing due to $\Delta R=1.4$ but only a small increase in LDR due to $\bar{R}=2.1$

\section{SUMMARY AND FUTURE WORK}

Using full 3D finite-element simulations of the cupping process for cylindrical and square cups, the features of the 1979 Hosford and 1989 Barlat 'TriComponent' yield surfaces are examined as compared to the $1948 \mathrm{Hill}$ tendencies to overpredict LDR and earing effects. The 89 Barlat seems to give results intermediate between the two others for earing phenomena, and agrees (in principle) exactly with 79 Hosford for normal anisotropy. In fact, a slight difference is observed which may be due to more accurate reproduction of the yield surface comers in the 89 Barlat implementation. For the case of earing in general and specifically with the D38 uranium examined here and in ref. [11], additional yield criteria such as the 6-component model of Barlat et al. [17] or other may be desired in the future.

\section{ACKNOWLEDGMENTS}

The author wishes to thank A.J. Sunwoo and D.H. Wood of LLNL for helpful discussions on the warm forming of uranium. This work was performed under the auspices of the U.S. Department of Energy by the Lawrence Livermore National Laboratory under contract W-7405-Eng-48.

\section{REFERENCES}

1. Wilson, D.V. and Butler, R.D., "The Role of CupDrawing Tests in Measuring Drawability", J. Inst. Metals 90, (1962) p. 473.

2. Logan, R.W., Meuleman, D.J., and Hosford, W.F., "The Effects of Anisotropy on the Limiting Drawing Ratio", in Formability and Metallurgical Structure, eds. A.K. Sachdev and J.D. Embury, TMS-AIME, (1987) p.159.

3. Yang, D.Y. and Kim, Y.J., "A Rigid-Plastic FiniteElement Formulation for the Analysis of General Deformation of Planar Anisotropic Sheet Metals and its Applications", Int. J. Mech. Sci. 28, No. 12, (1986) p. 825-840.

4. Logan, R.W., "Finite-Element Analysis of Earing Using Non-Quadratic Yield Surfaces", in NUMIFOAM 95 Proceedings, June 18-21, Ithaca, NY, ed. S.F. Shen and P.R. Dawson, Balkema publishers, Rotterdam, (1995) p.755.

5. Hayashida, Y., Maeda, Y., Matsui, K., Hashimoto, N., Hattori, S., Yanagawa, M., Chung, K., Barlat, F., Brem, J.C., Lege, D.J., and Murtha, S.J., "FEM analysis of punch stretching and cup drawing tests for aluminum alloys using a planar anisotropic yield function", in NUMIFORM 95 Proceedings, June 18-21, Ithaca, NY, ed. S.F. Shen and P.R. Dawson, Balkema publishers, Rotterdam, (1995) p.717.

6. Hill, R., The Mathematical Theory of Plasticity, Clarendon Press, Oxford (1948).
7. Hosford, W.F., "On Yield Loci of Anisotropic Cubic Metals", Proc. 7th N. Amer. Metal Working Research Conf., SME, Dearbom (1979) p. 191.

8. Whirley, R.G., and Hallquist, J.O., "DYNA3D: A Nonlinear, Explicit, Three-Dimensional Finite Element Code for Solid and Structural Mechanics- User Manual," University of California, Lawrence Livermore National Laboratory, Report UCRL-MA-107254 (1991).

9. Barlat, F. and Lian, J., "Plastic Behavior and Stretchability of Sheet Metals, Part I: A Yield Function for Orthotropic Sheets Under Plane Stress Conditions", int. J. Plasticity 5, (1989) pp. 51-66.

10. Kobayashi, S., Oh, S.I., and Altan, T., Metal Forming and the Finite-Element Method, Oxford Univ. Press, New York. (1979).

11. Hughes, A.N., Lane, R.A., and Orman, S., "The Effects of Warm Work on Some Mechanical and Fabrication Properties of Uranium", J. Nuclear Materials 48 (1973) p. 172.

12. Maker, B.N., "NIKE3D, A Nonlinear, Implicit, ThreeDimensional Finite Element Code for Solid and Structural Mechanics - User's Manual", UCRL-MA-105268, (1991).

13. Whirley, R.G., Engelmann, B.E., and Logan, R.W., "Some Aspects of Sheet Forming Simulation Using Explicit Finite Element Techniques", CED-Vol. 5, AMD Vol. 156, "Numerical Methods for Simulation of Industrial Metal Forming Processes", ed. M.J. Saran, A.B. Pifko, N. Kikuchi, and K.K. Tamma, ASME (1992).

14. Logan, R.W., and Hosford, W.F., "Upper-Bound Anisotropic Yield Locus Calculations Assuming [111] Pencil Glide", Int. J. Mechanical Sci. 22, (1980) p.419.

15. Krieg, R.D. and Key, S.W., "Implementation of a Time Dependent Plasticity Theory into Structural Computer Programs", Vol. 20 of Constitutive Equations in Viscoplasticity: Computational and Engineering Aspects, ASME, New York, NY, (1976) p.125.

16. Bathe, K.J., Finite Element Procedures in Engineering Analysis, Prentice-Hall, Englewood Cliffs, N.J. (1982).

17. Barlat, F., Chung, K., Shah, K.N., Lege, D.J. and Brem, J.C., "Modeling Sheet Forming of Polycrystaltine Metals", 2nd World Congress on Computational Mech., Stuttgart, Germany, Aug. 27-31, (1990) p. 383. 